\title{
Numerical analysis of $S O(10)$ models with flavour symmetries
}

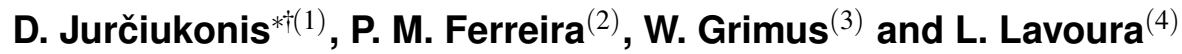 \\ (1) Vilnius University, Institute of Theoretical Physics and Astronomy \\ (2) Centro de Física Teórica e Computacional, Faculdade de Ciências, Universidade de Lisboa \\ (3) University of Vienna, Faculty of Physics \\ (4) CFTP, Instituto Superior Técnico, Universidade de Lisboa \\ E-mail: darius.jurciukonis@tfai.vu.lt
}

\begin{abstract}
We consider a supersymmetric $S O(10)$ Grand Unified Theory (GUT) in which the fermion masses are generated by renormalizable Yukawa couplings. Consequently, the scalar multiplets under consideration belong to the irreps $\mathbf{1 0}, \overline{\mathbf{1 2 6}}$, and $\mathbf{1 2 0}$ of $S O(10)$. We perform a complete investigation of the possibilities of imposing flavour symmetries in this scenario; the purpose is to reduce the number of Yukawa coupling constants in order to identify potentially predictive models. We have found that there are 14 inequivalent cases of Yukawa coupling matrices, out of which 13 cases pertain to one-generator Abelian groups and only one case has a two-generator symmetry group. Supersymmetry enters through the numerical examination of those cases, in which we have used the charged-fermion masses evaluated at the GUT scale through renormalization-group running in the context of the Minimal Supersymmetric Standard Model. However, the numerical analysis rules out almost all the cases, leaving only few viable ones which are compatible with the data on the fermion masses and mixings.

In order to test the viability of each case, and to find adequate numerical values for its parameters, we construct a minimization function $\chi^{2}$ which relate experimental data with the observables (masses and mixing parameters) to be fitted. For the numerical minimization we have employed the Differential Evolution algorithm. By modifying errors in the $\chi^{2}$ function and diversely restricting parameters space we have thus been able to test, for each case, more local minima, and to find the minima closer to the global minimum.
\end{abstract}

38th International Conference on High Energy Physics

3-10 August 2016

Chicago, USA

\footnotetext{
*Speaker.

${ }^{\dagger}$ D.J. thanks the Lithuanian Academy of Sciences for support through the project DaFi2016.
} 


\section{The model}

$S O(10)$ is a popular gauge group for the construction of Grand Unified Theories (GUTs). The reason is that its 16-plet accommodates at once all the chiral fields of one fermion family. However, inconsistencies in the fit of the experimental masses and mixings of the fermions led to the inclusion of the 120-plet; the resulting theory has been called [1] the "new minimal supersymmetric $S O(10)$ GUT" (NMSGUT).

However, adding a 120-plet leads to a proliferation of parameters in the Yukawa couplings; one might want to restrict the number of parameters in order to obtain potentially predictive scenarios [2].

The relevant fermion mass matrices are given by

$$
\begin{array}{ll}
M_{d}=k_{d} H+\kappa_{d} G+v_{d} F, & M_{u}=k_{u} H+\kappa_{u} G+v_{u} F, \\
M_{\ell}=k_{d} H+\kappa_{\ell} G-3 v_{d} F, & M_{D}=k_{u} H+\kappa_{D} G-3 v_{u} F,
\end{array}
$$

where $M_{d}, M_{u}$, and $M_{\ell}$ are the mass matrices of the down-type quarks, the up-type quarks, and the charged leptons, respectively, while $M_{D}$ is the neutrino Dirac mass matrix. The Yukawa-coupling matrices $H, G$, and $F$ are associated with the scalar irreps 10,120 , and $\overline{\mathbf{1 2 6}}$, respectively.

The coefficients $k_{d}, v_{d}, \kappa_{d}$, and $\kappa_{\ell}$ are the vacuum expectation values (VEVs) of the Higgs doublet components in the respective $S O(10)$ scalar irreps which contribute to the Higgs doublet $H_{d}$ of the Minimal Supersymmetric Standard Model (MSSM). The remaining coefficients (namely, $k_{u}, v_{u}, \kappa_{u}$, and $\kappa_{D}$ ) refer to $H_{u}$. The light-neutrino mass matrix is obtained as

$$
\mathscr{M}_{v}=M_{L}-M_{D} M_{R}^{-1} M_{D}^{\top}
$$

with $M_{L}=w_{L} F$ and $M_{R}=w_{R} F$, where $w_{L}$ and $w_{R}$ are the VEVs of the scalar triplets of the PatiSalam group $S U(4)_{c} \times S U(2)_{L} \times S U(2)_{R}$, which are a part of the scalar 126-plet of $S O(10)$.

The "Hermitian mass matrices" are diagonalized as

$$
\begin{array}{ll}
U_{d}^{\dagger}\left(M_{d} M_{d}^{\dagger}\right) U_{d}=\operatorname{diag}\left(m_{d}^{2}, m_{s}^{2}, m_{b}^{2}\right), & U_{u}^{\dagger}\left(M_{u} M_{u}^{\dagger}\right) U_{u}=\operatorname{diag}\left(m_{u}^{2}, m_{c}^{2}, m_{t}^{2}\right), \\
U_{\ell}^{\dagger}\left(M_{\ell} M_{\ell}^{\dagger}\right) U_{\ell}=\operatorname{diag}\left(m_{e}^{2}, m_{\mu}^{2}, m_{\tau}^{2}\right), & U_{v}^{\dagger}\left(\mathscr{M}_{v} \mathscr{M}_{v}^{\dagger}\right) U_{v}=\operatorname{diag}\left(m_{1}^{2}, m_{2}^{2}, m_{3}^{2}\right),
\end{array}
$$

where the matrices $U_{d, u, \ell, v}$ are unitary and $\left|m_{3}^{2}-m_{1}^{2}\right| \gg m_{2}^{2}-m_{1}^{2}>0$. The resulting fermion mixing matrices are

$$
V \equiv U_{\mathrm{CKM}}=U_{u}^{\dagger} U_{d}, \quad \text { and } \quad U_{\mathrm{PMNS}}=U_{\ell}^{\dagger} U_{v} .
$$

The neutrino mass spectrum is dubbed "normal" if $m_{3}^{2}>m_{1}^{2}$ and "inverted" otherwise.

Three important neutrino mass parameters are

$$
m_{\beta \beta} \equiv\left|\sum_{j=1}^{3} m_{j}\left[\left(U_{\mathrm{PMNS}}\right)_{1 j}\right]^{2}\right|, \quad m_{\text {tritium }} \equiv\left[\sum_{j=1}^{3} m_{j}^{2}\left|\left(U_{\mathrm{PMNS}}\right)_{1 j}\right|^{2}\right]^{\frac{1}{2}}, \quad m_{\mathrm{cosm} .} \equiv \sum_{j=1}^{3} m_{j} .
$$

Indeed, $m_{\beta \beta}$ is potentially measurable in experiments on neutrinoless double-beta decay, $m_{\text {tritium }}$ may be measured in experiments on the energy end-point of the beta decay of tritium, and $m_{\mathrm{cosm}}$. is an important quantity in the calculation of cosmological observables. 
Assuming that (a) all three matrices $H, F$, and $G$ are nonzero, (b) $\operatorname{det} F \neq 0$, and (c) there are no decoupled generations (experimental fact), we have obtained 14 inequivalent cases of Yukawa coupling matrices. 13 cases are generated by $\mathbb{Z}_{n}$ symmetries (with a suitable $n$ ), and one case is generated by the $\mathbb{Z}_{2} \times \mathbb{Z}_{2}$ symmetry [2].

\section{Numerical analysis}

The terms $M_{x} M_{x}^{\dagger}$ (where $x=d, \ell, u$ ) should fit 13 observables: nine charged-fermion masses and four observables in the CKM matrix. If considered, the term $\mathscr{M}_{v} \mathscr{M}_{v}^{\dagger}$ has to fit 5 additional parameters: three lepton mixing angles, the ratio $r_{\text {solar }}^{2} \equiv\left(m_{2}^{2}-m_{1}^{2}\right) /\left|m_{3}^{2}-m_{1}^{2}\right|$, and the value of $\left|m_{3}^{2}-m_{1}^{2}\right|$. We have used a fixed value $\left|m_{3}^{2}-m_{1}^{2}\right|=2.5 \times 10^{-15} \mathrm{MeV}^{2}$, which allows us to determine the overall scale of $\mathscr{M}_{v}, v i z .\left|v_{d} / w_{R}\right|$.

In order to test the viability of each case, and to find adequate numerical values for its parameters, we construct a $\chi^{2}$ function

$$
\chi^{2}(x)=\sum_{i=1}^{n}\left\{H\left[f_{i}(x)-\bar{O}_{i}\right]\left(\frac{f_{i}(x)-\bar{O}_{i}}{\delta_{+} O_{i}}\right)^{2}+H\left[\bar{O}_{i}-f_{i}(x)\right]\left(\frac{\bar{O}_{i}-f_{i}(x)}{\delta_{-} O_{i}}\right)^{2}\right\},
$$

where $n$ is the total number of the observables to be fitted (masses and the mixing parameters). Here $H$ is the Heaviside step function, $\bar{O}_{i}$ is the central value of each observable $O_{i}, \delta_{ \pm} O_{i}$ are the upper and lower errors of that observable, and $f_{i}(x), x=\left\{x_{\alpha}\right\}$, is the value of the observable in the probed case $x$. The data are fitted by minimizing $\chi^{2}(x)$ with respect to $\left\{x_{\alpha}\right\}$.

The values of the charged-fermion masses, renormalized at $M_{\mathrm{GUT}}=2 \times 10^{16} \mathrm{GeV}$, are taken from ref. [3]. The used values of the CKM mixing angles [4] are at low-energy scale; we have multiplied the error bars by a factor of three in order to obtain adequately large intervals. The neutrino-mixing observables are given by the $3 \sigma$ intervals from ref. [5].

In order to assess the fit feasibility of each case, we have first attempted to fit only the chargedfermion masses, then the charged-fermion masses together with the parameters of the CKM matrix, and, finally, all that together with the neutrino masses and the PMNS matrix parameters included. The total $\chi^{2}$ function is thus the sum of three terms: $\chi_{\text {total }}^{2}=\chi_{\text {masses }}^{2}+\chi_{\mathrm{CKM}}^{2}+\chi_{v}^{2}$. For the neutrino masses, we have analysed both possibilities of the normal and inverted neutrino mass spectra.

Finally, even if the value of $\chi_{\text {total }}^{2}$ could be reasonably low, we still had to check whether the ratio $\left|w_{R} / v_{d}\right|$ turned out in the right range. Indeed, since $v_{d}$ must be of order of the Fermi scale $100 \mathrm{GeV}$ and $w_{R}$ must be of order of the grand-unification scale $10^{16} \mathrm{GeV}$, we must require $\left|w_{R} / v_{d}\right|$ to be $10^{14}$ or even larger.

For the numerical minimization of the $\chi^{2}$ functions we have employed the Differential Evolution algorithm. This is a stochastic algorithm that exploits a population of potential solutions in order to effectively probe the parameter space. All the numerical calculations were implemented by using the programming language Fortran.

We have found that all the cases, except for the cases A and B, either fail to fit the observables adequately or give a too low value for $\left|w_{R} / v_{d}\right|$. Only the case $\mathrm{D}_{1}$ is able to fit all the observables, but all those good fits yield $\left|w_{R} / v_{d}\right|<3 \times 10^{13}$. 
We have found that the case B is able to fit perfectly all the observables. This is true irrespective of whether the neutrino mass spectrum is normal or inverted. However, when the neutrino mass spectrum is inverted the value of $\left|w_{R} / v_{d}\right|$ is too small.

For a normal neutrino mass spectrum in the case B, on the other hand, there are "fits" (sets of the parameters $\left\{x_{\alpha}\right\}$ ) in which all the inequalities, connected with the unification scale, are observed. The value of $\chi_{\text {total }}^{2}$ for the best fit is smaller than $10^{-3}$, i.e. for all practical purposes it is zero. The neutrino masses for this best fit are following: $m_{1} \approx 6 \times 10^{-3} \mathrm{eV}, m_{2} \approx 1 \times 10^{-2} \mathrm{eV}$, and $m_{3} \approx 5 \times 10^{-2} \mathrm{eV}$. One therefrom obtains $m_{\text {cosm. }} \approx 7 \times 10^{-2} \mathrm{eV}, m_{\text {tritium }} \approx 1 \times 10^{-2} \mathrm{eV}$, and $m_{\beta \beta} \approx 6 \times 10^{-3} \mathrm{eV}$.

The case A has much too many degrees of freedom, so it is adequate to try and constrain it somewhat. We follow a proposal of ref. [6], where real Yukawa-coupling matrices (due to an additional $C P$ symmetry) $F, G$, and $H$ are enforced and, moreover, $w_{L}=0$ is assumed, thereby discarding the type-II seesaw mechanism.

We have attempted to fit the case A both to the updated charged-fermion masses of ref. [3] and to the now extant value of $\sin ^{2} \theta_{13}$. We could achieve an excellent fit $\left(\chi_{\text {total }}^{2} \approx 0.005\right)$ when the neutrino mass spectrum is normal and a passable one $\left(\chi_{\text {total }}^{2} \approx 0.7\right)$ when the mass spectrum is inverted. The best fit with the normal hierarchy of the neutrino masses has $m_{\mathrm{cosm}} \approx 6 \times 10^{-2} \mathrm{eV}$, $m_{\text {tritium }} \approx 9 \times 10^{-3} \mathrm{eV}$, and $m_{\beta \beta} \approx 4 \times 10^{-3} \mathrm{eV}$. The best fit with the inverted hierarchy of the neutrino mass spectrum has $m_{\text {cosm. }} \approx 1 \times 10^{-2} \mathrm{eV}$ and $m_{\text {tritium }} \approx m_{\beta \beta} \approx 5 \times 10^{-3} \mathrm{eV}$.

Plots of fits to various observables and distributions of the neutrino masses for the cases A and $\mathrm{B}$ are presented in the poster of presentation [7].

\section{Conclusions}

The numerical analysis ruled out all 14 cases except for the case A and the case B. Both cases A and B allow excellent fits to the data when the neutrino mass spectrum is normal; when that spectrum is inverted, the case A can still fit the data but we were unable to find a good fit in the case B. Using parameters of fits we can make predictions for the neutrino sector. Finally, we conclude that within the new minimal supersymmetric $S O(10)$ GUT there are at most two possibilities to reduce the number of Yukawa couplings through flavour symmetries, while remaining in agreement with the data.

\section{References}

[1] C. S. Aulakh and S. K. Garg, Nucl. Phys. B 857 (2012) 101.

[2] P. M. Ferreira, W. Grimus, D. Jurčiukonis and L. Lavoura, Nucl. Phys. B 906 (2016) 289.

[3] Z. Z. Xing, H. Zhang, and S. Zhou, Phys. Rev. D 77 (2008) 113016.

[4] K. A. Olive et al. (Particle Data Group), Chin. Phys. C 38 (2014) 090001.

[5] D. V. Forero, M. Tórtola, and J. W. F. Valle, Phys. Rev. D 90 (2014) 093006.

[6] W. Grimus and H. Kühböck, Phys. Lett. B 643 (2006) 182.

[7] http://pos.sissa.it/archive/conferences/282/1187/ICHEP2016_1187_a1.pdf 